\title{
Influencia Interlingüística: Rasgos del Español en EI Criollo limonense
}

\author{
Argüello, René Zúñiga ${ }^{1}$ \\ Araya, Margie Cubillo ${ }^{2}$
}

\section{Resumo}

Depois da chegada dos primeiros jamaicanos à Costa Rica em 1872, a língua crioula trazida pelos trabalhadores, isto é o crioulo jamaicano, desenvolveu características únicas e distintivas após de mais de 140 anos. Alguns desses câmbios consumaram-se durante os primeiros 75 anos; entretanto o câmbio abrupto na língua dominante do inglês ao espanhol a partir de 1949 causou uma transformação mais radical. O fim deste artigo é justamente a descrição de alguns desses câmbios na fonologia e a morfossintaxe, os quais vêm sendo muito diferentes daqueles da língua original e que até poderiam ser atribuídos à influência do novo superstrato. Os fenômenos são tratados no marco das novas investigações em matéria de contato linguístico ao níveo sistemático em vez do níveo individual (SIEMUND, 2008).

Influência interlinguística. Nivel fonológico. Nivel morfosintático. Idiomas em contato.

Criollo limonense

\section{Resumen}

Tras la llegada de los primeros jamaiquinos a tierras costarricenses en 1872, la lengua criolla traída por los trabajadores (el criollo jamaiquino) desarrolló rasgos únicos y distintivos en más de 140 años de permanencia en el país. Este trabajo tiene como propósito describir ciertos fenómenos en niveles estructurales de la lengua que son distintos a los de aquella lengua inicial y que podrían ser atribuidos a la influencia del español, la lengua de superestrato con que se encontró el criollo proveniente de Jamaica.

Influencia interlingüística. Nivel fonológico. Nivel morfosintáctico. Lenguas en contacto. Criollo limonense

\footnotetext{
${ }^{1}$ René Zúñiga Argüello, Magister en Lingüística de la Universidad de Costa Rica, labora actualmente como profesor e investigador del programa PROLIBCA de la Universidad Nacional. Sus trabajos de investigación comprenden descripciones tipológicas del criollo limonense principalmente, así como trabajo en la revitalización de la lengua. Ha publicado y participado en eventos locales e internacionales, especialmente en el Caribe.

${ }^{2}$ Margie Cubillo Araya posee el grado de Magistra en Segundas Lenguas y Culturas de la Universidad Nacional de Costa Rica. Su enfoque investigativo comprende el estudio de metodologías y enfoques en la enseñanza de ESP, así como temas referentes a la interacción entre lenguas. Ha participado en congresos y simposios a nivel internacional
} 


\section{Introducción}

Tras la llegada de los primeros jamaiquinos a tierras costarricenses en 1872, la lengua criolla traída por los trabajadores (el criollo jamaiquino) desarrolló rasgos únicos y distintivos en más de 140 años de permanencia en el país. Este trabajo tiene como propósito describir ciertos fenómenos en niveles estructurales de la lengua que son distintos a los de aquella lengua inicial y que podrían ser atribuidos a la influencia del español, la lengua de superestrato con que se encontró el criollo proveniente de Jamaica. Los rasgos descritos a nivel fonológico son la pérdida de distinción entre fonemas en dos segmentos, así como la inclusión de una nueva realización alofónica para el fonema nasal alveolar. En cuanto a la morfología se describe el uso distinto de una prePosición de tiempo; mientras que a nivel sintáctico se analiza una estrategia de relativización distinta a la del criollo jamaiquino en las relaciones de genitivo y objeto de una comparación, además de la variación encontrada en los verbos ditransitivos que aceptan el orden: verbo, dativo, acusativo.

Este trabajo es el primer esbozo descriptivo de un conjunto de rasgos distintivos del Criollo limonense que pueden ser atribuidos al contacto con el español. El trabajo está organizado de la siguiente manera: en 2 se describen los fenómenos innovadores a nivel fonológico; en 3 a nivel morfológico; en 4 a nivel sintáctico. Los apartados anteriores se presentan con los rasgos sus realizaciones en criollo jamaiquino, inglés, español, y por último la innovación aparente en el Criollo limonense. Para finalizar, en 6 se sintetizan las conclusiones a la luz de posibles causas ya de naturaleza tipológica y o universal, o bien propias del fenómeno de contacto de lenguas. $\mathrm{Si}$, como podría ser el caso, los fenómenos en cuestión obedecen a una sustitución de acrolecto por superestrato, estaríamos ante un caso paradigmático en la el desarrollo de lenguas criollas, dado que estas: a) se relexifican, b) se estancan o desaparecen, o c) se mueven hacia el acrolecto. 


\section{Nivel Fonológico}

A nivel fonológico se observan tres fenómenos: pérdida de la oPosición entre el segmento fricativo labiodental sonoro /v/ y el oclusivo bilabial sonoro /b/; pérdida de la oPosición entre el segmento fricativo alveolar sordo /s/ y su contraparte sonora /z/, así como la realización velar del segmento nasal alveolar /n/

OPosición /b/, /v/

\section{CRIOLLO JAMAIQUINO}

El criollo jamaiquino cuenta con 21 consonantes fonémicas. En la descripción del sistema fonológico de Devonish y Harry (2004, p.264) y Harry (2006, p. 127) se encuentran los fonemas oclusivo bilabial sonoro /b/, y el fricativo labiodental sonoro /v/. En esta lengua se encuentran oposiciones entre ambos segmentos (1).

\section{Posición Inicial}
(1) /bet/ 'apostar'
/bwot/ 'bote'
vs /vet/ 'veterinario'
vs /vwot/ 'votar'

\section{INGLÉS}

El inglés cuenta con las mismas distinciones entre los fonemas oclusivo /b/ y fricativo /v/. En esta lengua se encuentran oposiciones entre ambos segmentos (2).

Posición Inicial

$\begin{array}{lll}\text { (2) } / \text { bet/ 'apostar' } & \text { vs } / \text { vet/ 'veterinario' } \\ \text { /bowt/ 'bote' } & \text { vs } / \text { vowt/ 'votar' }\end{array}$

\section{ESPAÑOL}

El español americano cuenta con 16 consonantes fonémicas, dentro de las cuales sólo se encuentra el fonema bilabial sonoro /b/ en todas las posiciones (3).

\section{Posición Inicial}

(3) /'baka/ 'vaca' 
Es importante recalcar que la ortografía española cuenta con las grafías $<b>$ y $<\mathrm{V}>$ aun cuando existe sólo el fonema /b/.

Para Martínez-Celdrán y otros (2003, p. 257) la grafía < v > en latín era solo una tipografía diferente para representar el fonema /u/; no existía diferencia alguna entre los fonemas /u/ y /v/ en cuanto a su ortografía. La /u/ breve tenía un alófono consonántico ante vocales que en la época clásica del latín correspondía al aproximante labial [w], pero luego pasó a pronunciarse como [ $\beta$ ].

Para representar este último sonido, ya en época moderna, se introdujo la grafía $<\mathrm{V}>$.

\section{CRIOLLO LIMONENSE}

El Criollo limonense cuenta con 21 consonantes fonémicas (ZÚÑIGA, 2012, p. 3). El fonema oclusivo bilabial sonoro /b/ aparece en el inventario de la lengua en todas las posiciones (4).
(4) /bed/ 'cama'
/ban/ 'nacer'

Asimismo, el segmento, fricativo labiodental sonoro /v/ aparece en todas las posiciones (5).
(5) /vjen/ 'vano'
/veks/ 'enojado'

Esta oPosición es distintiva en el Criollo limonense tanto como en el criollo jamaiquino; sin embargo, en el trabajo de campo con informantes, se ha notado una tendencia a adelantar el punto de articulación del segmento fricativo labiodental, para producir una realización oclusiva bilabial del mismo. Se percibe un uso cada vez más común del segmento oclusivo en Posición Inicial en palabras que deberían tener el segmento fricativo (6)

(6) ['beri] por /'veri/ 'muy'

Incluso, debido a esta pérdida de oPosición se han documentado, por medio de experimentos dirigidos, casos de homofonía en palabras que deberían considerarse como pares mínimos 


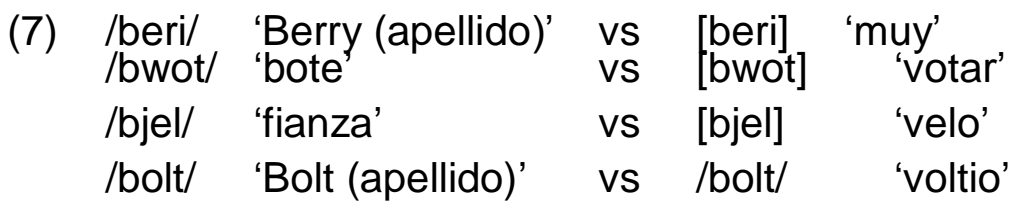

Ante la pérdida de oPosición fonemática en el CL en Posición Inicial y la preferencia por el segmento oclusivo bilabial, la influencia del nuevo superestrato puede trazarse ante la ausencia del segmento fricativo labiodental sonoro en el inventario fonológico del español.

OPosición /s/, /z/

\section{CRIOLLO JAMAIQUINO}

Según Devonish y Harry (2004, p. 264) y Harry (2006, p. 127) el criollo jamaiquino cuenta con los segmentos fricativo sordo /s/, y su contraparte sonora /z/. Ambas muestran oPosición en diversas posiciones (8-10)

Posición Inicial

(8) /su/ 'Sue (nombre)' vs /zu/ 'zoológico'

Posición media

(9) /'ljesi/ '(Lacey apellido)' vs /ljezi/‘vago'

Posición Final

(10) /prajs/ 'precio' vs /prajz/ 'premio'

\section{INGLÉS}

Al igual que el criollo jamaiquino, el inventario fonológico inglés tiene los fonemas /s/ y /z/. Ambos muestran oPosición en diversas posiciones (11-13)

Posición Inicial

(11) /su/ 'Sue (nombre)' vs /zu/ 'zoológico'

Posición media

(12) /'lejsi/ '(Lacey apellido)' vs /lejzi/‘vago’

Posición Final

(13) /prajs/ 'precio' Vs /prajz/ 'premio' 


\section{ESPAÑOL}

El español actual no presenta oPosición por sonoridad entre los sibilantes alveolares /s/ y /z/ desde el reajuste de sibilantes, fenómeno evolutivo que tuvo lugar entre el siglo XVI y el siglo XVII (CANO, 2005, p. 850). En el español actual, el fonema fricativo alveolar sordo /s/ aparece en todas las posiciones de palabra (14-16)

Posición Inicial

(14) /sal/ 'sal'

Posición media

(15) /'kasa/ 'casa'

Posición Final

(16) /lus/ 'luz'

\section{CRIOLLO LIMONENSE}

En el inventario consonántico del Criollo limonense (ZÚÑIGA, 2012, p.3) existe oPosición entre el fonema fricativo alveolar sonoro y el sordo. El segmento sordo /s/ aparece en todas las posiciones en la palabra (17-19).

Posición Inicial

(17) /sik/ 'enfermo'

/swop/ 'jabón'

Posición media

(18) /'isi/ 'fácil'

/'bisi/ 'ocupado'

Posición Final

(19) /tuma'tis/ 'tomate'

/fjes/ 'cara'

Asimismo, el segmento fricativo alveolar sonoro /z/ aparece en todas las posiciones (20-22).

Posición Inicial

(20) /zu/ 'zoológico'

Posición media

(21) /'rizn/ 'razón' 


\section{/'rjeza/ 'razurar'}

Posición Final

(22) /buz/ 'trago'

/dzaz/ 'jazz'

Sin embargo, en el trabajo con informantes se ha identificado la tendencia a ensordecer el segmento sonoro (23)

(23) [bi'kaas] por /bi'kaaz/ 'porque'

['rjesin] por /'rjezin / 'criar'

Al igual que los segmentos descritos anteriormente (oclusivo bilabial, fricativo labiodental), se evidencian casos en los que existe homofonía en donde debería haber pares mínimos (24).

(24) /su/ 'Sue (nombre)' vs [su] 'zoológico'

/'ljesi/ '(Lacey apellido)' vs ['ljesi] 'vago'

/prajs/ 'precio' vs [prajs] 'premio'

[sink]'precio' vs [sink]'premio'

Estos ejemplos muestran que la oPosición fonemática está siendo reemplazada por un solo fonema, en este caso el sordo. Como se mostró anteriormente, el español no cuenta con el fonema sibilante sonoro, lo cual puede estar causando la pérdida del fonema en todas las posiciones en el Criollo limonense.

\section{Velarización de $/ \mathbf{n} /$}

\section{CRIOLLO JAMAIQUINO}

En Devonish y Harry (2004, p. 264) y Harry (2006, p. 127) aparecen como fonemas los segmentos nasal alveolar $/ \mathrm{n} /$, así como el segmento nasal velar $/ \mathrm{\eta} /$. Ambas muestran oPosición en Posición Final (25).

$$
\text { /sin/ 'pecado' vs /sin/ 'cantar' }
$$

\section{INGLÉS}

El inglés muestra la misma oPosición fonemática en Posición Final (26). (26) $/ \mathrm{sin} / \quad$ 'pecado' vs $/ \mathrm{sin} /$ 'cantar' 


\section{ESPAÑOL}

El español no presenta oPosición fonemática entre los segmentos nasales alveolar $/ \mathrm{n} /$ y velar $/ \mathrm{h} /$. Sin embargo, en gran parte del español hispanoamericano, incluyendo el español de Costa Rica, el segmento /n/ se realiza como velar en Posición Final. Para Quesada (1991, p. 78), este rasgo fue diferenciador en tiempos de la colonia entre el habla del Noroeste y la del Valle Central; incluso, el autor menciona que todavía a inicios del siglo XX el rasgo era distintivo. Actualmente la realización velar del segmento nasal en Posición Final se ha extendido por todo el territorio costarricense (27) y es carácteristico de algunas variedades dialectales del español americano.

$\begin{array}{lll}\text { (27) } / \text { kan'sion/ } & \text { [kan'sion] } & \text { 'canción' } \\ \text { /me'lon/ } & \text { [me'lon] } & \text { 'melón' } \\ \text { /pe'on/ } & \text { [pion] } & \text { 'peón' }\end{array}$

\section{CRIOLLO LIMONENSE}

EI Criollo limonense tiene cuatro fonemas nasales (ZÚÑIGA, 2012, p. 3): bilabial $/ \mathrm{m} /$, alveolar /n/, palatal /n/ y velar /n/. Para este trabajo interesan los segmentos alveolar y velar. El segmento alveolar $/ \mathrm{n} /$ aparece en todas las posiciones de palabra (28-30).

Posición Inicial

(28) /nwo/ 'saber'

/najt/ 'noche'

Posición media

(29) /ka'nu/ 'canoa'

Posición Final

(30) /Jajn/ 'brillar' /rjen/ 'lluvia'

Por su parte, el segmento nasal velar / $\mathrm{h} /$ aparece cómo fonema sólo en Posición Final (31).

(31) /win/ 'ala' /sin/ 'cantar'

En el trabajo con informantes se ha identificado la velarización del segmento nasal alveolar en Posición Final (32) 
(32) [gaan] por /gaan/ 'irse'

[bon] por /bon/ 'quemar'

[baan] por /baan/ 'nacer'

['uman] por /'uman/ 'nacer'

Bajo experimentos dirigidos con informantes, se ha observado velarización de segmentos nasales alveolares en

Posición Final, lo cual elimina pares mínimos existentes (33).

(33) /sin/ 'cantar' vs [sin] 'pecado'

/tin/ 'cosa' vs [tin] 'lata'

Los datos muestran una velarización del segmento alveolar en Posición Final, tal y como sucede en el español de Costa Rica. Este fenómeno se da en el superestrato en todo el territorio nacional, lo cual pudo haber influido a iniciar el fenómeno.

\section{Nivel Morfológico}

A pesar de existir indicios de influencia del español en algunas estructuras morfológicas como lo identifica Winkler (1998, p.1) en la combinación de raíces lexicales españolas con afijos del Criollo limonense, este apartado describe únicamente el morfema funcional in (prePosición de tiempo) en el Criollo limonense.

\section{Preposiciones: in y an \\ CRIOLLO JAMAIQUINO}

La lengua distingue dos usos para las preposiciones de tiempo in 'en', y pan 'en'. La prePosición in tiene exclusividad de uso para espacios temporales prolongados, por ejemplo años, meses, estaciones, etc. (34)

(34) Dem kom in Aagos

3SG venir en agosto

'Ellos vinieron en agosto'

Mi byebi ban in 2009

1SG bebé nacer en 2009

'Mi bebé nació en 2009'

In di somataym

en DET verano

'En el verano' 
De igual forma, el uso de la prePosición pan está restringido a días y fechas específicas (35).

(35) Jumyeka get independent pan aagast 6 in 1962 Jamaica obtenerindependiente en agosto 6 en1962 'Jamaica se independizó el 6 de agosto de 1962'

Existen casos en que el hablante elide la prePosición pan, especialmente delante de días de la semana (36).

(36) Shi go chorch sondye

3SG ir iglesia domingo

'Ella fue a la iglesia el domingo'

\section{INGLÉS}

La lengua distingue dos usos para las preposiciones de tiempo in 'en', y on 'en'. La prePosición in tiene exclusividad de uso para espacios temporales prolongados, por ejemplo años, meses, estaciones, etc (37).

(37) Theycame in August

3SG venir en agosto

'Ellos vinieron en agosto'

Mi baby was born in 2009

1SG bebé COP nacer en 2009

'Mi bebé nació en 2009'

De igual forma el uso de la prePosición on está restringido a días y fechas específicas, tal y como sucede en el criollo jamaiquino (38).

(38) Kid-s have to go to school on Monday niño-PL tener INF ir a escuela en lunes

'Los niños tienen que ir a la escuela el lunes'

\section{ESPAÑOL}

El español cuenta con la prePosición en que denota el tiempo en que se determina una acción, por lo general se utiliza en periodos extendidos de tiempo, por ejemplo estaciones, meses, años, siglos, etc. (39).

(39) Ellos vinieron en agosto

Él nació en 1998

Llueve mucho en invierno 
Por otra parte, para periodos de tiempo más específicos como días o fechas, el español no usa prePosición para relacionar los elementos. (40).

(40) Tengo clase los jueves

Costa Rica se independizó el 15 de setiembre de 1821

Ella va a misa los domingos

\section{CRIOLLO LIMONENSE}

Las preposiciones in 'en' y an 'en' muestran misma distribución en el criollo jamaiquino (in y pan) y en el Criollo limonense. La prePosición in se utiliza para periodos de tiempo extendidos como años, meses, estaciones, etc (41).

(41) Bab Maali ban in 1945

Bob Marley nacer en 1945

'Bob Marley nació en 1945'

Di kaanival iz in aktaba

DET carnaval COP en octubre

'El carnaval es en octubre'

Uno berdye iz in di syem mont

2PL cumpleaños COP en DET mismo mes

'El cumpleaños de ustedes es en el mismo mes'

Al igual que en el criollo jamaiquino, se utiliza la pre Posición an (pan en el criollo jamaiquino) para relacionar elementos a días y fechas específicas (42). A diferencia del criollo jamaiquino, no se han registrado casos de elisión de la prePosición an, en ningún contexto.

(42) Shi go chorch an sondye

3SG ir iglesia en domingo

'Ella fue a la iglesia el domingo'

A pesar de esta distribución en el uso de ambas preposiciones, se nota un uso distinto de la prePosición para relacionar elementos con un tiempo específico, e.g. fechas y días. En estos casos, se registra una tendencia hacia el uso de in

(43) A si mi onkl in krismos

1SG ver 1SG tío en Navidad

'Vi a mi tío en Navidad'
A waz
ban
in aagast 31 in
1983
1SG COP.ANT nacer en agosto 31 en 1983
'Nací el 31 de agosto de 1983' 
A wil go owt in di wikend

1SG FUT ir afuera en DET fin de semana

'Voy a salir en el fin de semana'

Es importante anotar que, a pesar de que el uso de una prePosición no es necesario en el español cuando se trata de relacionar elementos con un tiempo específico, el uso de la prePosición in en el Criollo limonense puede derivarse del contacto con el español.

Las preposiciones de tiempo del inglés in, on y at tienen como equivalente directo en español a la prePosición en. Este aumento en la distribución respecto del español causa interferencia i.e. en los en los hablantes nativos de español que aprenden el inglés como segunda lengua al producir oraciones como en (44).

(44) * I was born in August 31st, 1983
1SGCOP.ANT nacer en agosto 31ero 1983
'Nací el 31 de agosto de 1983'

Si bien es cierto las lenguas criollas tienden a poseer una prePosición general para expresar locación o tiempo (HOLM, 1988, p. 208), la tendencia a utilizar la prePosición in, y no otra, en el Criollo limonense se marca por la influencia del español.

\section{Nivel Sintáctico}

A nivel sintáctico se han descrito fenómenos producidos por la interferencia lingüística del español, e.g. cambio en el orden de constituyentes en la frase nominal Winkler (1998, p.1). Este apartado trata dos fenómenos de influencia sintáctica: estrategia de la retención de pronombre en cláusulas relativas para las relaciones de genitivo y objeto de una comparación, y verbos ditransitivos que aceptan el dativo en segunda Posición .

\section{Estrategia de retención de pronombre en cláusulas relativas CRIOLLO JAMAIQUINO}

El criollo jamaiquino posee tres estrategias de representación de la FNRel, a saber espacio vacío, pronombre relativo y retención de pronombre (Patrick, 2003, p.23). Estas estrategias permiten relativizar todas las relaciones gramaticales de la jerarquía de Keenan y Comrie (1977, p. 66). Para efectos de 
este trabajo, interesan únicamente las relaciones de genitivo y objeto de una comparación debido a que, como se muestra más adelante, son las únicas que presentan una estrategia de representación diferente en el Criollo limonense. Christie (1996 apud PATRICK, 2003, p.24), describe el uso de dos estrategias de representación para la relación de genitivo: a) el uso del pronombre relativo huufa 'cuyo' (45), y b) la estrategia de retención de pronombre (46).

(45) Di uman huufa biebi dem tiif gaan a stieshan DET mujer REL bebé 3PL robar irse a estación 'La mujer cuyo niño robaron el niño se fue a la estación'

(46) Di uman we dem tiif ar biebi gaan a stieshan DET mujer REL 3PL robar 3SG bebé irse a estación 'La mujer que le robaron el niño se fue a la estación'

En el caso de la relación gramatical de objeto de una comparación, la única estrategia válida es la del pronombre relativo (47).
A da man
de mi taal-a
dan
COP DET hombre allá 1SG alto-COMP que
'Ese es el hombre que yo soy más alto que él'

\section{INGLÉS}

El inglés estándar posee dos estrategias de representación de la FNRel, espacio vacío y pronombre relativo. En el caso del genitivo y objeto de una comparación se prefiere el uso de la estartegia de pronombre relativo (48).
(48) The woman whose house is red ha-s a daughter DET mujer REL casa COP rojo tener-3SG DET hija
'La mujer cuya casa es roja tiene una hija'
The woman whose baby they stole went to the station DET mujer REL bebé 3PL robarirse a DET estación 'La mujer que le robaron el niño se fue a la estación'

En el caso de la relación gramatical de objeto de una comparación, el inglés tiene ambas estrategias, y además es agramatical el uso de la estrategia de retención de pronombre
(49) That is the man
who I am tall-er than
DEM COP DET hombre REL 1SG COP alto-COMP que
'Ese es el hombre que yo soy más alto que él' 


\section{ESPAÑOL}

El español cuenta con la estrategia del pronombre relativo para representar la FNRel, estos pronombres son: que, el cual (y sus variaciones la cual, los cuales, las cuales, lo cual), quien, cuyo, como, donde, cuando y cuanto. Al relativizar la oración subordinada en la relación del genitivo, el español hace uso del pronombre relativo cuyo (50).

(50) Me encontré al viejo cuyo hijo es compañero del mío.

La persona cuyo vehículo es placas 324455, por favor preséntese al parqueo.

Sin embargo, el uso de este pronombre es cada vez menor en el habla real, Elvira (2007, p 374) describe como "un retroceso progresivo, que resulta patente en su frecuente sustitución por otras construcciones alternativas". Esa construcción alternativa a la que se refiere Elvira es el uso de la estrategia de retención de pronombre para la relación de genitivo en la que se sustituye el cuyo por el pronombre relativo que y un posesivo (51).

(51) Me encontré al viejo que su hijo es compañero del mío.

La persona que su vehículo es placas 324455, por favor preséntese al parqueo. Respecto de la relación gramatical de objeto de una comparación, el español cuenta únicamente con la estrategia de retención de pronombre para poder identificar el rol del antecedente en la cláusula relativa (52). De no ser por esta estrategia, la relativización de esta relación sería imposible.

(52) Ese es el hombre que yo soy más joven que él

Ahí va mi compañera que yo me saque más nota que ella

\section{CRIOLLO LIMONENSE}

Al igual que el criollo jamaiquino, el Criollo limonense posee tres estrategias de representación de la FNRel: espacio vacío, pronombre relativo, y retención de pronombre. No obstante, hay que recalcar que las instancias en donde se utiliza la retención de pronombre como estrategia son en relativizaciones que implican las relaciones del genitivo (53) y de objeto de una comparación (54).

$\begin{array}{lllll}\text { Di uman we ar ozban jumyekan kom yesidye. } & \text { we } \\ \text { DET mujer REL } 3 S G \text { esposo jamaiquino venir } & \text { ayer } \\ \text { 'La mujer que el esposo es jamaiquino vino ayer' } & \end{array}$


Di bway

we dem kot im fut de lok op ina di owz

DET muchacho REL 3PL cortar 3SG pi ! COP encerrar en DET

casa

'El hombre que le cortaron el pie está encerrado en la casa'

(54)

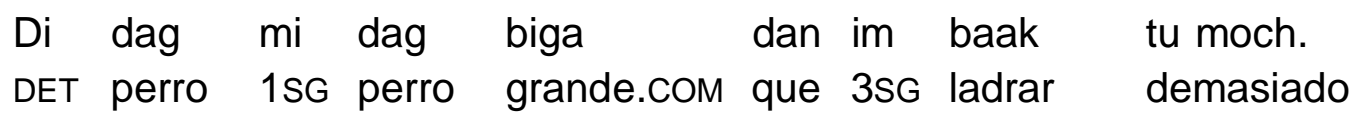

'El perro que mi perro es más grande que él ladra demasiado'

Dat iz di man uu ay faas-a dan im.

DEM COP DET hombre REL 1SG rápido-COMP que 3SG

'Ese es el hombre que yo soy más rápido que él'

Si bien es cierto el criollo jamaiquino cuenta con la estrategia de retención de pronombre para genitivos al igual que el Criollo limonense, se puede argumentar que el criollo jamaiquino cuenta también con un pronombre para esta relación (huufa 'cuyo'), el cual es inexistente el Criollo limonense. La pérdida de esta estrategia hace pensar en la influencia del nuevo superestrato ya que esta estrategia es la de mayor uso actualmente en el español.

Adicionalmente a la influencia del español, el uso exclusivo de la estrategia de retención de pronombre en las relaciones de genitivo objeto de una comparación pueden relacionarse con la premisa propuesta por Lehmann (2010, p. 10), en la que considera que la relación gramatical relativizada no es de tanta importancia como lo es el grado de nominalización de la cláusula relativa:

Representation of the head in various syntactic functions in the external-head relative clauses correlates inversely with the degree of nominalization of the relative clause; i.e., the more strongly nominalized a relative clause is, the less it will allow of pronominal representation of the head in the relativized position.

Esta afirmación se refleja en el uso de la estrategia más explícita del Criollo limonense (retención de pronombre) para relativizar las relaciones menos nominalizadas de la escala (genitivo y objeto de una comparación). 


\section{El dativo en verbos ditransitivos INGLÉS}

En inglés existen ciertos verbos ditransitivos que no permiten el orden verbo, dativo acusativo. En su lugar el uso de la prePosición to 'a' debe introducir el dativo (55)

(55) She explain-ed that to me.

3SG explicar-ANT DEM a 1SG

'Ella me explicó eso'

Así, sin la introducción de to ante el dativo con verbos como explain, describe, suggest, donate, y otros más, la oración sería agramatical (56)

(56) * She explain-ed me that.

3SG explicar-ANT 1SG DEM

'Ella me explicó eso'

\section{CRIOLLO JAMAIQUINO}

El criollo jamaiquino tiene dos alternativas en su orden de constituyentes en oraciones ditransitivas. En Posición post verbal: verbo, dativo, acusativo; o bien verbo acusativo $y$ frase preposicional introduciendo al dativo por medio del morfema funcional $t u$. Al igual que el inglés, algunos verbos (eksplyen, diskrayb, donyet, sajes, etc.) solo admiten el segundo orden (57), siendo el primer orden un caso de agramaticalidad (58).

(57) Shi sajes di plyes tu mi.

3SG sugerir DET lugar a 1SG

'Ella me sugirió el lugar'

(58) * Shi sajes mi di plyes.

3SGsugerir 1SG DET lugar

'Ella me sugirió el lugar'

\section{ESPAÑOL}

La ditransitividad en el español por su parte, da una Posición pre verbal a los clíticos que preceden un verbo conjugado manteniendo el orden dativo, acusativo (59)

(59) Eso me lo explicó Juan ayer

Es una buena película, te la sugiero

Esa tierra se la donó el gobierno a los Arias 
Esta Posición pasa a ser post verbal con un verbo infinitivo o imperativo positivo, estructuras en las que se mantiene el orden dativo acusativo sin excepción (60)

(60) Explíqueme eso otra vez.

Es una buena película, jsugiérasela!

Esa tierra, el gobierno quería donársela a los Arias.

\section{CRIOLLO LIMONENSE}

En la gran mayoría de los casos, el Criollo limonense utiliza el mismo orden de constituyentes en oraciones ditransitivas que el criollo jamaiquino. En otras palabras, Posición post verbal con dos alternativas: verbo, dativo, acusativo (61); o bien verbo acusativo y frase preposicional introduciendo al dativo por medio del morfema funcional tu (62).

(61) Shi gi mi di moni.

3SG dar 1SG DET plata

'Ella me dio la plata'

Myeri sen im di leta yesidye

María enviar 3SG DET carta ayer

'María le envió la carta ayer'

(62) Shi gi di moni tu mi.

3SG dar DET plata a 1SG

'Ella me dio la plata'

Myeri seen di leta tu im yesidye

María enviar DET carta a 3SG ayer

'María le envió la carta ayer'

De manera interesante, el Criollo limonense no discrimina verbos en el cambio de orden de los constituyentes. Bajo experimentos dirigidos se pusieron a prueba los verbos que restringen el uso del orden verbo, dativo, acusativo en el criollo jamaiquino y en el inglés. Los resultados demuestran que el Criollo limonense trata esos verbos como cualquier otro de la lengua. El orden verbo, dativo, acusativo (63), y verbo, acusativo, dativo introducido por prePosición (64) es gramatical con todos los verbos ditransitivos del Criollo limonense.

(63) Shi eksplyen mi dat.

3SG explicar 1SG DEM

'Ella me explicó eso'

Go plis diskrayb dem di rum!

IR.IMP porfavor decribir.IMP 3SG DET cuarto 
'¡Vaya por favor, descríbales el cuarto!'

$\mathrm{Mi}$ mada donyet $\mathrm{mi}$ dis pis a laan

1SG madre donar 1SG DEM pieza de tierra

'Mi mamá me donó este pedazo de tierra'

(64) Shi eksplyen dat tu mi.

3sG explicar dem a 1SG

'Ella me explicó eso'

Go plis diskrayb di rum tu dem!

IR.IMP porfavor decribir.IMP DET cuarto a 3SG

'¡Vaya por favor, descríbales el cuarto!'

$\mathrm{Mi}$ mada donyet dis pis a laan tu mi

1SG madre donar dem pieza de tierra a 1SG

'Mi mamá me donó este pedazo de tierra'

Esta distribución de los constituyentes de las oraciones ditransitivas muestra similitud con el español, claro está que esta similitud se da por medio del orden post verbal que poseen las cláusulas con verbos en infinitivo y en imperativo en español.

\section{Conclusiones}

Tras haber analizado los hallazgos, los fenómenos en los que se observa una innovación aparentemente producida por el contacto del Criollo limonense con el español son:

- La pérdida de o Posición fonemática entre los segmentos /b/, /v/ y /s/, /z/.

- La realización velar [n] del segmento nasal alveolar /n/ en Posición Final de palabra.

- El uso de la prePosición in (equivalente directo de la prePosición española 'en' en el Criollo limonense) para referir tiempos específicos.

- La estrategia de retención de pronmbre como relativizador exclusivo para las relaciones del genitivo y objeto de una comparación

- La posibilidad de utilizar el orden verbo, dativo, acusativo en oraciones ditransitivas para todos los verbos.

- La influencia estructural del español se manifiesta en distintos fenómenos que dan características propias al Criollo limonense. 


\section{Referências}

CANO, Rafael (coord.) Historia de la lengua española. 2 ed. Madrid: Ariel, 2005. $1168 p$.

DEVONISH, Hubert; HARRY; Otelemate. "Jamaican Creole and Jamaican English: Phonology", en KORTMAN, B; SHNEIDER E. W., A Handbook of Varieties of English: Fonología 1. Berlin: Mouton De Gruyter, 2004. 1226 p.

ELVIRA, Javier. Observaciones sobre el uso y la diacronía de cuyo Homenaje a Ramón Santiago, Ediciones del Orto, Madrid, v 1, p 365 - 375, 2007.

GIVÓN, Talmy. Syntax: An introduction. Vol. II. Amsterdam: John Benjamins, 2001. $554 p$.

HARRY, Otelemate G. Jamaican Creole. Journal of the International Phonetic Association, Cambridge Journals, Alemania, v. 36, n. 1, p. 125-131., 2006

HERZFELD, Anita. Mekaytelyu. La lengua criolla. San José: Editorial de la Universidad de Costa Rica, 2002.

HOLM, John. Pidgins and creoles. Cambridge: Cambridge University Press, 1988. $257 p$.

KEENAN, Edward; COMRIE, Bernard. Noun phrase accessibility and universal gramar. Linguistic Inquiry, MIT Press, Massachusetts, v. 8, n. 1, p. 63-99. Invierno, 1977.

LEHMANN, Christian. On the typology of relative clauses. Linguistics

An Interdisciplinary Journal of the Language Sciences, Mouton De Gruyter, Berlin, p. 663-680, 2010.

MARTÍNEZ-CELDRÁN, et al. Castilian Spanish Journal of the International Phonetic Association, Cambridge Journals, Alemania, v. 33, n. 2, p. 255-259, 2003

PATRICK, Peter. (2003). Jamaican creole morphology and syntax. Colchester, Inglaterra: University of Essex. Disponible en: <http://privatewww.essex.ac.uk>. Accesado el 22 de setiembre de 2011.

PAYNE, Thomas. Describing morphosyntax. Cambridge: Cambridge University Press, 1997. 413p.

QUESADA, Miguel Ángel. El español de Guanacaste. 1 ed. San José: Editorial Universidad de Costa Rica, 1991. 235p.

WINKLER, Elizabeth. Limonese Creole: A case of contact-induced language change. 1998. Tesis doctoral. Bloomington: Indiana University, 1998.

ZÚÑIIGA, René. Fonología del Criollo limonense. En SIMPOSIO INTERNACIONAL DE ACALING, 1, Heredia: Universidad Nacional. 2012 